\title{
Tecnologías de la Información y Comunicaciones
}

\section{Information and Communication Technologies}

José Luis Monteagudo Peña

Área de Investigación en Telemedicina y Sociedad de la Información y Departamento de Informática Sanitaria de la Escuela Nacional de Sanidad del Instituto de Salud Carlos III. Madrid

Las Tecnologías de la Información y las Comunicaciones constituyen una herramienta fundamental para soportar las actividades profesionales de investigación en biomedicina y salud. Su adopción y uso está ligado a la eficiencia y competitividad.

Tras el análisis de las necesidades se han identificado las aplicaciones TICs cuyo dominio es recomendable. Las necesidades se refieren al desarrollo de las actividades propias de los proyectos de investigación pero también de formación continuada, docencia y desarrollo profesional.

Sobre la base anterior, se propone una estructura de programa formativo basado en niveles y con una mezcla de bases generales comunes de tipo horizontal y áreas de especialización vertical.

Finalmente, se pone de manifiesto que las nuevas tecnologías además de facilitar nuevos instrumentos significan nuevas culturas de trabajo y presentan nuevos dilemas de carácter ético y legal para los investigadores que necesitarán formarse en nuevos entornos de trabajo.

Palabras clave: Investigación biomédica, Tecnologías de la Información y Comunicaciones, Internet, e-Salud, Telemedicina
Information and Communication Technologies are essential to support professional research activities in biomedicine and health. Their adoption and use is linked to efficiency and competitivity.

After need analysis, TICs applications whose knowledge is recommended, are identified. Needs are referred to activities linked to the execution of research projects but also to teaching, continuous training and professional development.

Based on that, it is proposed a formative programme structure with different competence levels and with a combination of horizontal common general skills and vertical specialization areas.

Finally, it is outlighted that new technologies facilitate new instruments but also they represent new working cultures and present new ethic and legal dilemmas to the researchers that would need to be educated in new working environments.

Keywords: Biomedical research, Information and Communication Technologies, Internet, e-Health, Telemedicine

\footnotetext{
Correspondencia:

José Luis Monteagudo Peña

Departamento de Informática Sanitaria

Escuela Nacional de Sanidad

Instituto de Salud Carlos III

C/Sinesio Delgado 8

Madrid

E-mail: jlm@isciii.es
}

\section{INTRODUCCIÓN}

Las Tecnologías de la Información y las Comunicaciones (TICs) son hoy día instrumentos básicos para la investigación biomédica y de salud. La adopción y el uso eficiente de las TICs están ligados a la capacidad competitiva de los investigadores y las organizaciones en un marco de globalización de la ciencia ${ }^{1,2}$. 
Los investigadores constituyen un prototipo de "trabajadores del conocimiento". Su actividad es intensiva en el manejo de información, la obtención y el procesamiento de datos. La comunicación con otros colegas, el trabajo cooperativo en grupo y la difusión de los resultados constituyen otros elementos característicos de la actividad investigadora que cada vez se sustenta más en el uso de tecnologías de la información y comunicaciones.

El rango de uso de estas tecnologías es muy amplio cubriendo desde redes de sensores y microdispositivos electrónicos al diseño de fármacos con supercomputadores, o el uso de laboratorios automatizados y biotelemetría de pacientes ambulatorios.

La popularización de los ordenadores personales ha supuesto la posibilidad de disponer de capacidades crecientes de soportes de ofimática, de cálculo matemático y bioestadística.

Por otra parte, la investigación biomédica y de salud ha tenido vocación de colaboración internacional para la difusión de resultados y para el desarrollo de proyectos multicéntricos. Hoy día, gracias a Internet, los investigadores tienen acceso a una fuente ingente de información en la punta de sus dedos en su propia mesa y, además, es posible el trabajo en grupos, consorcios, y redes independientemente de la localización de los investigadores o los recursos de conocimiento. Así por ejemplo, tanto el Programa Nacional de I+D+I en España como el Programa Marco de la UE han promovido las redes temáticas multicéntricas de investigación.

Un aspecto importante a considerar es la velocidad de cambio de la tecnología así como su extensión creciente en todas las actividades. Esta proliferación plantea también cuestiones de especialización y eficiencia así como adaptación a la velocidad de cambio. Algunas preguntas son: ¿qué conocimientos debe poseer un investigador concreto?, ¿qué materias se deben dominar y con qué profundidad?, ¿cual es la forma más apropiada de adquirirlos?, ¿cómo incorporar las capacidades tecnológicas para los centros investigadores? ¿cómo compaginar grupos multidisciplinares integrando expertos tecnológicos y biomédicos?

Los investigadores tradicionalmente se forman dentro de grupos de investigación junto a otros colegas más experimentados de los que aprenden técnicas y el uso de equipos y dispositivos. Se trata de procesos de formación continua que muy frecuentemente y más de lo deseable descansan en el esfuerzo personal y autodidacta. La cuestión de la capacitación de los investigadores se ha visto tradicionalmente como una cuestión de "mérito personal" y por lo tanto dejado fundamentalmente a la iniciativa individual.

Hoy día la investigación biomédica y de salud es (o debería ser) también un aspecto estratégico para las organizaciones sanitarias y los estados. Por si misma constituye un renglón económico importante y cada vez más está orientada a la optimización del uso de recursos y la obtención de resultados evaluables.

Las nuevas tecnologías presentan un carácter dual, ya que si bien por una parte hacen posible que investigadores y laboratorios tengan acceso a capacidades impensables apenas hace unos años, por otra parte son un factor de ampliación potencial de la brecha entre los que disponen de las nuevas tecnologías y la capacidad de usarlas y los que no ${ }^{3}$.

Hay que tener en cuenta que la formación en metodologías de la investigación relacionadas con las nuevas tecnologías tiene que considerar no solamente aspectos técnicos sino, lo que quizá es más importante, nuevos entornos y nuevas formas de trabajar ${ }^{4}$. Lógicamente la situación es muy distinta según las organizaciones y sus enfoques. En algunos centros de investigación se han incorporado ingenieros biomédicos o técnicos informáticos formando equipos multidisciplinares. No obstante, los profesionales de la investigación sanitaria deben tener un mínimo de conocimientos sobre uso de las nuevas tecnologías para poder desarrollar su actividad. En los párrafos siguientes se identifican los conocimientos y habilidades deseables para los investigadores de Biomedi-cina y Salud en relación con las Tecnologías de la Información y Comunicaciones. Sobre esta base se hacen recomendaciones para el contenido curricular dentro de la formación en metodología de la investigación, que se estructura con diferentes niveles y componentes para satisfacer los requisitos de diferentes perfiles de investigador.

No se trata de presentar un programa detallado, sino más bien de un marco de referencia general y flexible.

\section{IDENTIFICACIÓN DE NECESIDADES DE CONOCIMIENTOS DE TICS PARA INVESTIGACIÓN BIOMÉDICA Y DE SALUD}

La formación de un investigador debería estar optimizada para poder abordar de la mejor forma posible las tareas que debe afrontar en su trabajo ${ }^{5}$. En cada caso existirá un nivel de exigencia diferente dependiendo del tipo de investigación, las tareas específicas y la organización en que se desarrolla. Es 
muy distinto para un contexto de investigación clínica que para ingeniería biomédica o telemedicina.

Se pueden distinguir niveles de conocimientos sobre TICs, horizontales comunes a todo tipo de investigador, como consecuencia de la existencia de un marco de actividades genéricas comunes. Básicamente estas son:

- Ejecución de tareas técnicas y científicas propias de la realización de un proyecto de investigación

- Adquisición de información, nuevos conocimientos y habilidades

- Transmisión de conocimiento a través de actividades de docencia en cursos, seminarios, dirección de tesis doctorales

- Trabajos propios de gestión de la investigación, tales como la participación en comités y grupos de expertos nacionales e internacionales; evaluación de proyectos para agencias financiadoras

- Actividades de relaciones profesionales, incluyendo la participación en asociaciones profesionales y científicas; organización de congresos; dictado de conferencias; y evaluación de artículo para revistas científicas; difusión en prensa y en otros medios.

- Además, existen otros aspectos de conocimientos necesarios, no estrictamente técnicos relacionados con los nuevos entornos de trabajo y culturas organizativas que propician las nuevas tecnologías.

A continuación se describen con más detalle las actividades y las aplicaciones TICs siguiendo la clasificación anterior. En la Tabla 1 se ofrece un esquema con la correspondencia entre actividades y uso TIC.

\section{SOPORTES Y APLICACIONES TICS} RELACIONADAS CON LA EJECUCIÓN DE PROYECTOS DE INVESTIGACIÓN

Un proyecto incluye tres fases principales: a) concepción, preparación y presentación de una propuesta, b) realización de las tareas de investigación programadas, y c) materialización de los resultados mediante publicación de resultados, realización de patentes, redacción de informes y tareas de difusión general.

\section{a) Herramientas y soportes TIC para}

la realización de una propuesta de Proyecto

Actividades típicas correspondientes a esta fase son la recopilación de información; búsquedas bibliográficas; realización de documentos (memorias, CVs); diseño de planes de trabajo; cálculos de presupuestos y comunicación con otros investigadores participantes en la propuesta.

Las tecnologías TIC que soportan estas tareas incluyen el acceso a Internet, correo electrónico navegación WWW y eventualmente de acceso a ficheros (ftp). También son muy útiles las herramientas de acceso a bases de datos documentales y bibliográficas para el soporte documental.

Se hace necesario dominar los paquetes llamados de ofimática, es decir, aquellos que incluyen procesador de textos, editor de gráficos y hojas de cálculo. Más allá de estas aplicaciones es muy conveniente el uso de herramientas para la gestión de proyectos que permiten la descripción detallada de las tareas, los recursos y su articulación en el tiempo con diagramas de tipo Gantt o PERT.

Cada vez más, los proyectos se realizan por grupos de investigadores y consorcios de diferentes organizaciones a menudo internacionales. En estas situaciones son de gran utilidad las herramientas de trabajo colaborativo en grupo.

\section{b) Realización tareas de investigación}

La realización de un proyecto de investigación comporta tareas que son comunes a todos ellos tales como: recopilación de información; realización de informes y memorias; seguimiento del plan de trabajo; gestión económica-administrativa y relación con otros miembros del equipo de investigación.

Para estos fines se utilizan herramientas TIC similares a las observadas en el apartado anterior tales como procesadores de texto; hojas de cálculo; trasparencias y gráficos; gestor de proyectos; servicios de Internet incluyendo correo electrónico, navegación WWW, ftp, y soportes para trabajo cooperativo en grupo.

Además de estas actividades generales existe una gran variedad de aplicaciones de las TIC en tareas de investigación biomédica dependiendo de cada tipología de investigación. Las TICs se aplican en las tareas científicas y técnicas propias de la realización de los proyectos de investigación tanto para el diseño y montajes experimentales como para la adquisición de datos en experimentos de laboratorio o de campo, así como su trasmisión, almacenamiento, procesado, recuperación y visualización. Los datos pueden consistir en anotaciones de texto, variables alfanuméricas, señales biológicas, sonidos o imágenes dependiendo de cada caso.

Para la adquisición manual de datos se puede hacer uso de terminales portátiles digitales o PDAs, Palms, Tablet PCs, Lap tops que pueden incluir sistemas de localización e información geográfica (GPS, GIS) y comunicaciones inalámbricas de datos vía radio o por GPRS usando la red celular. 
Tabla 1: Resumen de las actividades de investigación en biomedicina y salud y las aplicaciones TICs relacionadas

\begin{tabular}{|c|c|}
\hline Actividades Investigación & Requisitos conocimientos sobre TICs \\
\hline \multicolumn{2}{|l|}{ 1. Realización de propuestas de proyectos } \\
\hline $\begin{array}{l}\text { Recopilación de información } \\
\text { Realización documentos: Memoria Plan de trabajo } \\
\text { Presupuesto } \\
\text { Comunicación con otros investigadores y agencias financiadoras }\end{array}$ & $\begin{array}{l}\text { Acceso Internet. e-mail. Navegación WWW. ftp. } \\
\text { Acceso bases de datos documentales y bibliográficas. } \\
\text { Edición documentos. Procesador de Textos. Editor de Gráficos. } \\
\text { Hoja de cálculo } \\
\text { Gestión de Proyectos } \\
\text { Herramientas trabajo cooperativo }\end{array}$ \\
\hline \multicolumn{2}{|l|}{$\begin{array}{l}\text { 2. Realización tareas técnicas y científicas de un proyecto de investigación } \\
2.1 \text { Aplicaciones generales }\end{array}$} \\
\hline $\begin{array}{l}\text { Recopilación de información } \\
\text { Realización documentos } \\
\text { Gestión de proyecto } \\
\text { Comunicación y trabajo cooperativo intra e intergrupos } \\
\text { Comunicación con agencia financiadora }\end{array}$ & $\begin{array}{l}\text { Acceso Internet. } \\
\text { e-mail. Navegación wwW. } \\
\text { Acceso bases de datos documentales y bibliográficas } \\
\text { Edición documentos. Procesador de Texto. Editor de Gráficos. } \\
\text { Hoja de cálculo } \\
\text { Paquete Gestión de Proyectos } \\
\text { Herramientas soporte trabajo cooperativo en grupo }\end{array}$ \\
\hline \multicolumn{2}{|l|}{ 2.2 Aplicaciones específicas } \\
\hline $\begin{array}{l}\text { Toma manual de datos de laboratorio y de campo } \\
\text { Medidas experimentales } \\
\text { Adquisición, transmisión y almacenamiento de datos } \\
\text { Procesado de datos, señales e imágenes }\end{array}$ & $\begin{array}{l}\text { PDAs; Palms; Lap-tops; terminales digitales } \\
\text { Instrumentación computarizada } \\
\text { Automatización de laboratorios } \\
\text { Robótica y teleactuación, Sistemas optoelectrónicos, Redes de datos } \\
\text { Buses de instrumentación, Biotelemetría y telemedicina } \\
\text { Paquetes matemáticos y de estadística } \\
\text { Paquetes proceso de señales biomédicas } \\
\text { Estaciones de trabajo gráficas }\end{array}$ \\
\hline
\end{tabular}

2.3 El ordenador y la Red como laboratorios de investigación

Modelado y simulación

Generación de imágenes

Realización de mapas y análisis basados en distribución geográfica

Soportes a la toma de decisiones

Genómica, Biología molecular, Biología estructural

Síntesis de fármacos

Herramientas modelado matemático

Modelado de sistemas, procesos y estructuras

Sistemas de realidad virtual, Estaciones de trabajo gráficas

Sistemas imágenes 3D

Sistemas de localización (GPS) e información geográfica (GIS)

Inteligencia artificial, Bioinformática y Biología computacional

Supercomputación, GRID, Web semántico

Agentes inteligentes de red, Inteligencia Ambiental

3. Materialización Resultados Proyectos

Publicaciones, informes,

Patentes

Difusión

Comunicación con editores y medios

Procesador de Texto. Editor de Gráficos. Hoja de cálculo. Dibujo.

Foto digital

Acceso a bases de datos documentales y bibliográficas

Presentaciones proyección PC

Realización CD-ROMs. Edición video Edición páginas Web (html)

e-mail. Navegación www

4. Formación continua. Adquisición conocimientos

Formación continua

Trabajo cooperativo en grupo para edición documentos

5. Docencia

Docencia presencial y electrónica

Acceso Internet

Herramientas e-learning

Acceso información científica y documental

Visualizadores multimedia

Presentaciones gráficas

Herramientas de autor para cursos virtuales

Creación páginas web (html)

Uso plataformas gestión conocimiento

6. Gestión de la investigación

Participación en comités y grupos de expertos nacionales

e internacionales.

Evaluación de proyectos para agencias financiadoras.

Evaluación de artículos para revistas científicas

Acceso Internet, e-mail

Navegación www

Herramientas de ofimática

\section{Actividades profesionales}

Participación en asociaciones científicas

organización de congresos, conferencias,

artículos revistas generales y prensa

Acceso Internet. e-mail, Navegación www

Herramientas de ofimática

\section{Aspectos no tecnológicos} Seguridad

\section{Encriptado}

Firma electrónica

Protección virus

Análisis de riesgos y medidas protección

Estándares ISO; CEN; HL7

Interoperabilidad

Codificación, terminología, Historia Clínica

Electrónica; DICOM; Medical bus

Linux; HTML; XML 
En los entornos de investigación en laboratorio o clínica los trabajos de investigación descansan en instrumentación electrónica que cada vez más está computarizada trabajando en red y con buses de instrumentación. La automatización de laboratorios y los sistemas robotizados son una realidad creciente en áreas relevantes de investigación biomédica, mientras los sistemas de biotelemetría y de telemedicina representan recursos s técnicos que se utilizan en diferentes modelos de investigación clínica.

Cada línea de investigación presenta perfiles de uso de dispositivos electrónicos y recursos informáticos muy diversos, aunque en general se puede observar que la tendencia es hacia un aumento de sistemas computerizados, conectados en red y con automatización de las tareas de adquisición transmisión y procesados de la información.

Cada vez cobra mayor relevancia la utilización del ordenador y la red (internet) como auténticos "laboratorios de investigación". En este contexto se deben contemplar los trabajos que descansan en la explotación intensiva de bases de datos; análisis de imágenes o registros biomédicos; simulación de procesos; síntesis de compuestos farmacéuticos o modelado de biomoléculas.

Para estas actividades se deben dominar recursos de computación avanzada; supercomputación; herramientas de adquisición, procesado, trasmisión y visualización de de imágenes y registros biológicos; análisis matemático y estadístico; estaciones gráficas de alta capacidad etc. En esta línea se deben considerar los avances que se están produciendo en agentes inteligentes, Web semántico o computación GRID. Otro aspecto importante lo constituyen las herramientas para el desarrollo de modelos y de simulación de sistemas así como todo el capítulo de inteligencia artificial y de soporte a la toma de decisiones.

\section{c) Soportes TIC para la materialización \\ de los resultados de investigación}

Básicamente se trata de actividades relacionadas con la publicación y difusión de resultados científicos. Esto significa la producción y edición de documentos y materiales gráficos incluyendo contenidos multimedia, foto digital, video, CD ROMs, DVDs, PáginasWeb, y audio y video-streaming.

\section{SISTEMAS TIC PARA SOPORTE DE FORMACIÓN CONTINUA Y DOCENCIA}

Tal como se ha comentado anteriormente los investigadores requieren el acceso a información y mantienen una formación continua a lo largo de su vida profesional. En este sentido, aparte de las técnicas tradicionales, cada vez se utilizan más las posibilidades que ofrece Internet para formación a distancia (e-learning) y acceso a documentación.

Las TICs sustentan capacidades de modelos y simulación, incluyendo técnicas de realidad virtual que soportan aprendizajes avanzados.

Por otra parte los investigadores, en general, desarrollan actividades de formación y trasferencia de conocimientos impartiendo cursos o tutelando becarios y nuevos investigadores. A este fin se deben contemplar las herramientas de autor para la generación de materiales didácticos, así como para la producción de cursos, tutoría de alumnos y la gestión usando Internet.

Cada vez cobran más importancia los sistemas de "gestión del conocimiento" que soportan bases documentales compartidas dentro de una organización y herramientas de búsquedas sistemáticas de recursos.

\section{SOPORTES TICS PARA OTRAS ACTIVIDADES}

En este apartado se incluyen las correspondientes a la gestión de la investigación y de actividades profesionales. Entre las primeras se contemplan actividades tales como la participación en comités y grupos de expertos nacionales e internacionales así como la evaluación de proyectos para agencias financiadoras o la participación como experto en misiones científicas.

Por otra parte los investigadores están involucrados en actividades profesionales participando en asociaciones científicas; consejos editoriales de publicaciones; comités organizadores de congresos; dictando conferencias, y desarrollando actividades de difusión general.

Para sustentar estas actividades se utilizan recursos que fundamentalmente son medios de comunicación, agendas electrónicas; correo electrónico; navegación www y aplicaciones de ofimática. En este ámbito son muy importantes las funciones de "oficina móvil".

\section{CUESTIONES RELACIONADAS CON USO DE TIC}

El desarrollo de la TICs representa no solo instrumentos para soporte a la realización de las tareas de investigación sino que también afecta a la forma en que se realizan los procesos y las relaciones entre personas e instituciones. Su uso implica aspectos éticos y legales que deben tenerse en cuen- 
Tabla 2. Diferentes niveles de conocimiento requeridos en TICs para los investigadores de biomedicina y salud

\begin{tabular}{l|l}
\hline Nivel & Conocimientos \\
\hline N0 & Conocimientos mínimos necesarios \\
\hline N1 & Conocimientos básicos generales \\
\hline N2 & Conocimientos para líneas de investigación por especialidad (epidemiología, clínica, biomolecular, farmacia, fisiología, etc.) \\
\hline N3 & Conocimientos específicos alto nivel de especialización \\
\hline N4 & Conocimientos líneas intensivas TIC (Ingeniería biomédica, telemedicina, e-salud, bioinformática) \\
\hline
\end{tabular}

ta por los investigadores. Entre ellos se encuentran los requisitos de protección de datos, confidencialidad y seguridad. Otro tipo de cuestiones legales se refiere a la propiedad intelectual en medios electrónicos y la cuestión de patentabilidad.

Además, la normalización en las TICs es una cuestión muy importante que está ligada a la interoperabilidad de equipos y sistemas. La normalización se refiere ciertamente a las redes y a los elementos físicos, pero también a la codificación de datos médicos, comunicación de registros de señales o imágenes médicas y a la arquitectura de historias clínicas y mensajería electrónica en salud.

El mundo de la investigación biomédica también está inmerso en el debate sobre el uso de sistemas abiertos frente a sistemas propietarios. Es importante conocer las posibilidades y ventajas del llamado software libre (open source), cuya referencia principal lo constituye el sistema operativo Linux. Aparte de las consideraciones técnicas existen factores de concepción de la comunidad científica, trasferencia de conocimiento y acceso a la tecnología de gran relevancia para la cooperación científica.

\section{PROPUESTA DESARROLLO CURRICULAR}

Tal como ya hemos comentado, el tipo de actividades de investigación en medicina y salud es muy amplio y se desarrollan en diversos marcos organizativos. Dependiendo de cada especialidad, tipo de investigación y actividad concreta se requerirá un perfil de conocimiento y destreza diferentes. Son distintas las herramientas y la profundidad de conocimientos que se requieren para abordar una investigación en Epidemiología que para Telemedicina o Genómica y son muy diferentes los requerimientos en un equipo multidisciplinar con alta especialización de funciones en un centro de investigación de alto nivel que para un investigador solo o en un equipo reducido en una institución de un país en desarrollo.
Teniendo en cuenta estos hechos, se pueden distinguir diferentes niveles de exigencia de dominio tecnológico, tal como se muestra en la Tabla 2.

El nivel de conocimiento " 0 " se refiere a conocimientos previos mínimos que requiere un profesional para desenvolverse en el sector. Incluiría el uso básico de PC y de aplicaciones de ofimática tales como procesador de texto, hoja de calculo, editor de gráficos y presentaciones de diapositivas. También se requiere familiaridad en el uso de correo electrónico y navegación por la www en Internet.

El nivel 1 se refiere a conocimientos básicos generales recomendables para todo profesional dedicado a la $\mathrm{I}+\mathrm{D}+\mathrm{I}$ en biomedicina y salud. Contenidos de este nivel sería uso de herramientas para acceso a bases de datos documentales y bibliográficas; paquetes de bioestadística; aplicaciones para gestión de proyectos; conocimientos básicos de redes y sobre sistemas de adquisición, archivo y proceso de datos. Deseable conocimiento de herramientas para trabajo cooperativo en grupo.

Un nivel superior, nivel 2, se situarían los conocimientos de materias TICs con niveles de intensificación deseables para un investigador en un área concreta de "especialización" biomédica. Existirán materias que pueden ser comunes a varias especialidades y otras no. Por ejemplo, un investigador en neurofisiología deberá tener conocimientos sobre análisis digital de señales biológicas que no son necesarios para un investigador en salud pública.

Finalmente se tienen que considerar los conocimientos sobre temas concretos específicos de alta especialización (Nivel 3)

Un caso especial lo componen las necesidades de conocimientos para investigadores en áreas intensivas en TICs para la salud como son Ingeniería biomédica, e-Salud, telemedicina ó bioinformática. (Nivel 4)

El conocimiento de herramientas de edición multimedia, páginas web y sistemas de e-Learning podrían constituir un modulo complementario espe- 
Fig. 1. Diagrama mostrando la estructura modular propuesta por niveles de conocimiento y por especialización.

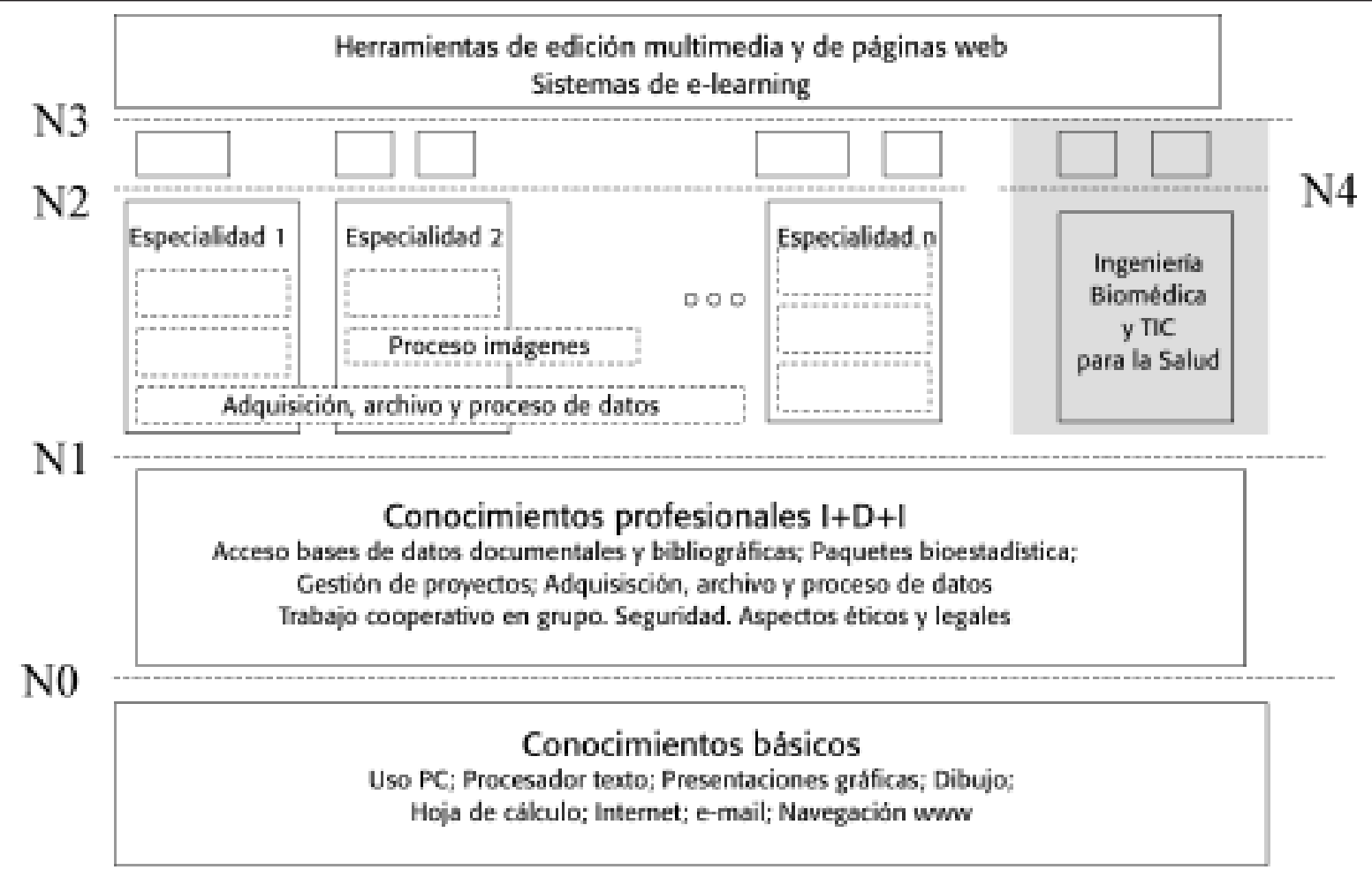

cialmente enfocado a investigadores involucrados o con interés en formación a distancia

La Fig. 1 muestra de forma esquemática la estructura general para el desarrollo modular de una formación adecuada a diferentes perfiles de demanda sobre conocimientos TICs de investigadores biomédicos y de salud

\section{RESUMEN Y CONCLUSIONES}

La creciente difusión de las aplicaciones de las tecnologías de la información y las comunicaciones en la sociedad actual se hace especialmente patente en la investigación biomédica y de salud. Las nuevas tecnologías se hacen presentes en la instrumentación y equipamiento electrónico pero también para sustentar la adquisición, manejo y difusión de la información científica. El carácter global de las ciencias de la salud se soporta hoy día gracias a Internet que ha devenido imprescindible en el trabajo cotidiano de los investigadores no solo para las tareas de realización de los proyectos sino también para su formación continua, la trasferencia de sus conocimientos, la relación con otros colegas y las actividades profesionales.

Las nuevas tecnologías ofrecen nuevas capacidades ampliadas pero simultáneamente presentan el riesgo de aumentar la brecha entre los que tienen acceso a ellas y los que no. La principal barrera es el conocimiento y dominio de su uso. Aparte de las cuestiones técnicas existen elementos de adaptación a una nueva cultura de trabajo. La rápida evolución de las TICs. obliga a considerar formatos de formación continua, flexible y a distancia.

Por otra parte, las tecnologías son una herramienta y no un fin en si mismas. Su conocimiento debe entenderse en el contexto de idoneidad y utilidad. En este trabajo se ha partido de la identificación de necesidades en función de las tareas que debe realizar un investigador. Se ha planteado un esquema de conocimientos por niveles y perfiles de especialidad.

El dominio de la cultura de las TICs involucra no solamente aspectos técnicos sino también cuestiones relacionadas con su selección, estándares, cuestiones éticas y legales, propiedad intelectual, y software libre.

Por supuesto las instituciones de investigación y las autoridades deben tener en cuenta la importancia estratégica de dotar de infraestructuras tecnológica y muy especialmente la conectividad a Internet y para gestión del conocimiento. Las capacidades de investigación en biomedicina y salud dependerán en 
ultimo término de las capacidades de los investigadores para incorporar y hacer uso de los avances tecnológicos en su trabajo cotidiano.

\section{REFERENCIAS}

1. Hasman A et al. Education and Training in Health Informatics in Europe; IOS Press, Amsterdam, 1995.

2. Mantas, J. (Ed.) Health Telematics Education; IOS Press, Amsterdam, 1997
3. Rodríguez, R J; Oliveri, N C; Monteagudo, JL; Hernández, A; Sandor, T "e-Salud en Latinoamérica y el Caribe. Tendencias y Temas Emergentes”. Organización Panamericana de la Salud. OMS. Marzo 2003. Pp.1-208.

4. Monteagudo, J.L. “Telemedicina”. Informática y Salud. No 29. Enero-Febrero 2001. pp1499-1502

5. Guillén C, Monteagudo JL "Priorities for Health Professionals in Education and Training on Information Technology: results of a Delphi study" Health Telematics Education. J. Mantas (Ed.) IOS Press Amsterdam 1997, pp 51-60.

\section{Sesión de trabajo}

Prof. A. Castell, Prof. F. Llamosas, Prof. C. Noldin, Prof. I. Rodríguez y Prof. L. Spitale

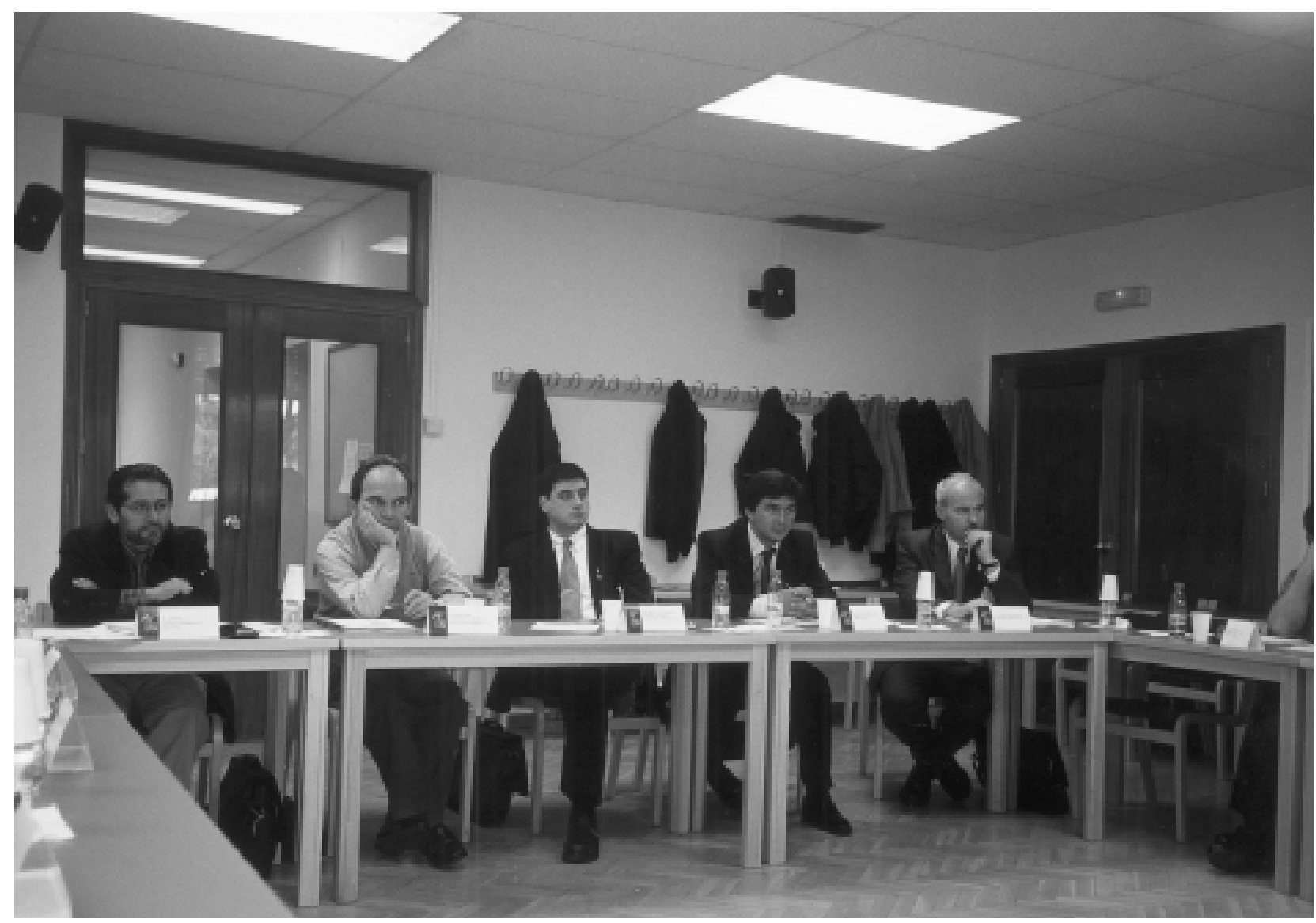

